\title{
Finescale Structure of the $T-S$ Relation in the Eastern North Atlantic
}

\author{
R. FERRARI \\ Massachusetts Institute of Technology, Cambridge, Massachusetts \\ K. L. POLZIN \\ Woods Hole Oceanographic Institution, Woods Hole, Massachusetts
}

(Manuscript received 26 April 2004, in final form 10 February 2005)

\begin{abstract}
Distributions of temperature $(T)$ and salinity $(S)$ and their relationship in the oceans are the result of a balance between $T-S$ variability generated at the surface by air-sea fluxes and its removal by molecular dissipation. In this paper the role of different motions in setting the cascade of $T-S$ variance to dissipation scales is quantified using data from the North Atlantic Tracer Release Experiment (NATRE). The NATRE observational programs include fine- and microscale measurements and provide a snapshot of $T-S$ variability across a wide range of scales from basin to molecular. It is found that microscale turbulence controls the rate of thermal dissipation in the thermocline. At this level the $T-S$ relation is established through a balance between large-scale advection by the gyre circulation and small-scale turbulence. Further down, at the level of intermediate and Mediterranean waters, mesoscale eddies are the rate-controlling process. The transition between the two regimes is related to the presence of a strong salinity gradient along density surfaces associated with the outflow of Mediterranean waters. Mesoscale eddies stir this gradient and produce a rich filamentation and salinity-compensated temperature inversions: isopycnal stirring and diapycnal mixing are both required to explain the $T-S$ relation at depth.
\end{abstract}

\section{Introduction}

The establishment of the temperature-salinity $(T-S)$ relationship in midlatitudes has concerned oceanographers for nearly a century. Wüst (1935) pointed out that "the vertical structure of the Subantarctic Intermediate Water, with its horizontal spreading at depths, is analogous to a vertical figure of the horizontal arrangement of temperature and salinity at the surface of the formation region." Iselin (1939) further noted that the correspondence was between horizontal profiles in the winter mixed layer and vertical profiles in the thermocline and concluded that thermocline waters must be derived from surface waters in winter. This picture is incomplete because the $T-S$ relationship does not remain unchanged as waters are transported along surfaces of constant density. Some other process acts on $T-S$ properties as they flow into the ocean interior. Iselin (1936)

Corresponding author address: Raffaele Ferrari, MIT Bldg. 54, Rm. 1420, Department of Earth, Atmospheric, and Planetary Sciences, 77 Massachusetts Ave., Cambridge, MA 02139-4307.

E-mail: rferrari@mit.edu argued that the tightness and linearity of the $T-S$ curve in the western North Atlantic Ocean implicates a dominant balance between horizontal advection by largescale flows and diapycnal mixing. Montgomery (1938) and Iselin (1939) argued for a dominant balance between isopycnal advection by the large-scale circulation and isopycnal stirring by small-scale eddies. In this paper we use data from the North Atlantic Tracer Release Experiment (NATRE) to quantify the processes that establish the $T-S$ relationship in the North Atlantic.

The $T-S$ relationship in the midlatitude thermocline is now understood as being set at the surface by thermohaline forcing in the form of convective production of mode waters (McCartney 1977) and subduction of winter mixed layers (Stommel 1979). The water mass properties, that is, points on the $T-S$ curve (Sverdrup et al. 1942), are then advected into the interior by the mean ocean circulation, dispersed by eddies, and modified by diapycnal mixing. The combined effect of isopycnal stirring and diapycnal mixing is to make water parcels warmer and saltier, and to increase their potential vorticity, as they flow into the interior (McCartney 1982). We have learned much about isopycnal (Rob- 
bins et al. 2000) and diapycnal (Ledwell et al. 1998) processes in the past 70 years, but the implications of these observations for the establishment of the $T-S$ relationship have not been fully appreciated. There is a dichotomy between the effect of stirring and mixing on water mass properties. Diapycnal mixing tends to homogenize different water types and consequently tighten the $T-S$ relation. Isopycnal stirring creates contrasts between water types and enhances variability in the $T-S$ relation. This is simply the distinction between stirring and mixing of Eckart (1948) applied to the $T-S$ diagram. The contribution of this manuscript is to quantify the roles of eddy stirring and diapycnal mixing in creating the $T-S$ relation in the eastern North Atlantic from an analysis of $T-S$ variability. The analysis is based on standard techniques developed for turbulence studies. The novelty is that we have three scales to worry about: the mean circulation, mesoscale motions including geostrophically balanced eddies and internal waves, and three-dimensional turbulence.

The paper is organized as follows. Vertical profiles and mooring measurements collected as part of the NATRE experiment are described in section 2. In section 3, we use qualitative arguments to demonstrate that along-isopycnal eddy advection plays a major role in shaping the $T-S$ relationship at the Mediterranean waters level. To be more quantitative, in section 4, we introduce a triple decomposition of variables into mean, meso-, and microscales. The triple decomposition is used to analyze observations and quantify the rates of along-isopycnal and cross-isopycnal transports, for buoyancy in section 5 and for temperature in section 6. In section 7, we provide discussion and conclusions.

\section{Observations}

\section{a. Water mass properties}

The eastern subtropical North Atlantic was the subject of an intensive series of field programs in 1991-93 as part of the North Atlantic Tracer Release Experiment (Ledwell et al. 1998) and the Subduction Experiment (Joyce et al. 1998). We use fine- and microstructure data obtained from the former and current-meter data from the latter to characterize and quantify the processes shaping the $T-S$ relation. Time mean velocities are to the southwest in the upper thermocline (Schott and Stommel 1978; Ledwell et al. 1998) with weak velocities in the main thermocline and below. The mesoscale eddy variability is atypically weak for the North Atlantic (Zang and Wunsch 2001) with rms subinertial velocities of less than $3 \mathrm{~cm} \mathrm{~s}^{-1}$. Ledwell et al.
(1998) and St. Laurent and Schmitt (1999) diagnose diapycnal downwelling at $10 \%$ of the Ekman pumping velocity (R. A. Weller et al. 2004, unpublished manuscript). Diapycnal dispersion rates are "weak," $K_{\rho} \cong 1-2$ $\left(\times 10^{-5} \mathrm{~m}^{2} \mathrm{~s}^{-1}\right)$, in the upper thermocline and increase slightly with depth (Toole et al. 1994). Isopycnal dispersion rates are somewhat smaller than $K_{e} \cong 1 \times 10^{3}$ $\mathrm{m}^{2} \mathrm{~s}^{-1}$, as we show in section 6 .

The variability we seek to describe in the NATRE data is generated by stretching and folding the basinscale water mass properties through isopycnal stirring and diapycnal mixing. The North Atlantic subtropical thermocline consists largely of North Atlantic Central Water (NACW). This water mass is convectively formed in the northeastern part of the subtropical gyre (McCartney 1982; Siedler et al. 1987) and is characterized by weak isopycnal gradients. These gradients typically coincide with large-scale patterns in hydrological forcing and late winter/early spring mixed layer properties (Stommel 1979). An exception to this rule is that NACW is saltier in the eastern North Atlantic than in the west. Mauritzen et al. (2001) find that this trend is associated with detrainment of Mediterranean Outflow Water in the eastern Gulf of Cadiz; that is, the trend is generated by mixing processes in the ocean interior. Immediately beneath the thermocline, contrasts between saline Mediterranean Water (MW) and fresher Antarctic Intermediate Water and Labrador Sea Water result in strong $T-S$ gradients along isopycnal surfaces (Fig. 1). In this paper we show that these gradients are stirred by the weak mesoscale eddy field in the NATRE region and support the creation of a rich finescale $T-S$ structure.

\section{b. High-resolution profiler and current-meter data}

Distributions of $T$ and $S$, and their relationship, are the result of a balance between the thermocline variability generated by large-scale motions and its removal by molecular dissipation. To address the disparate range of scales involved, we use data from the highresolution profiler (HRP) and standard current-meter data.

The HRP is a free-falling, internally recording vertical profiler (Schmitt et al. 1988; Polzin and Montgomery 1996). Relative velocities are measured with an acoustic velocimeter. Profiles of oceanic velocity are computed from relative velocity, accelerometer, and magnetometer data using a variation of the Total Ocean Profiling System model developed by Hayes et al. (1984). Temperature, conductivity, and pressure are sensed with an NBIS Mark III CTD. The HRP also carries a microstructure suite consisting of two airfoil 


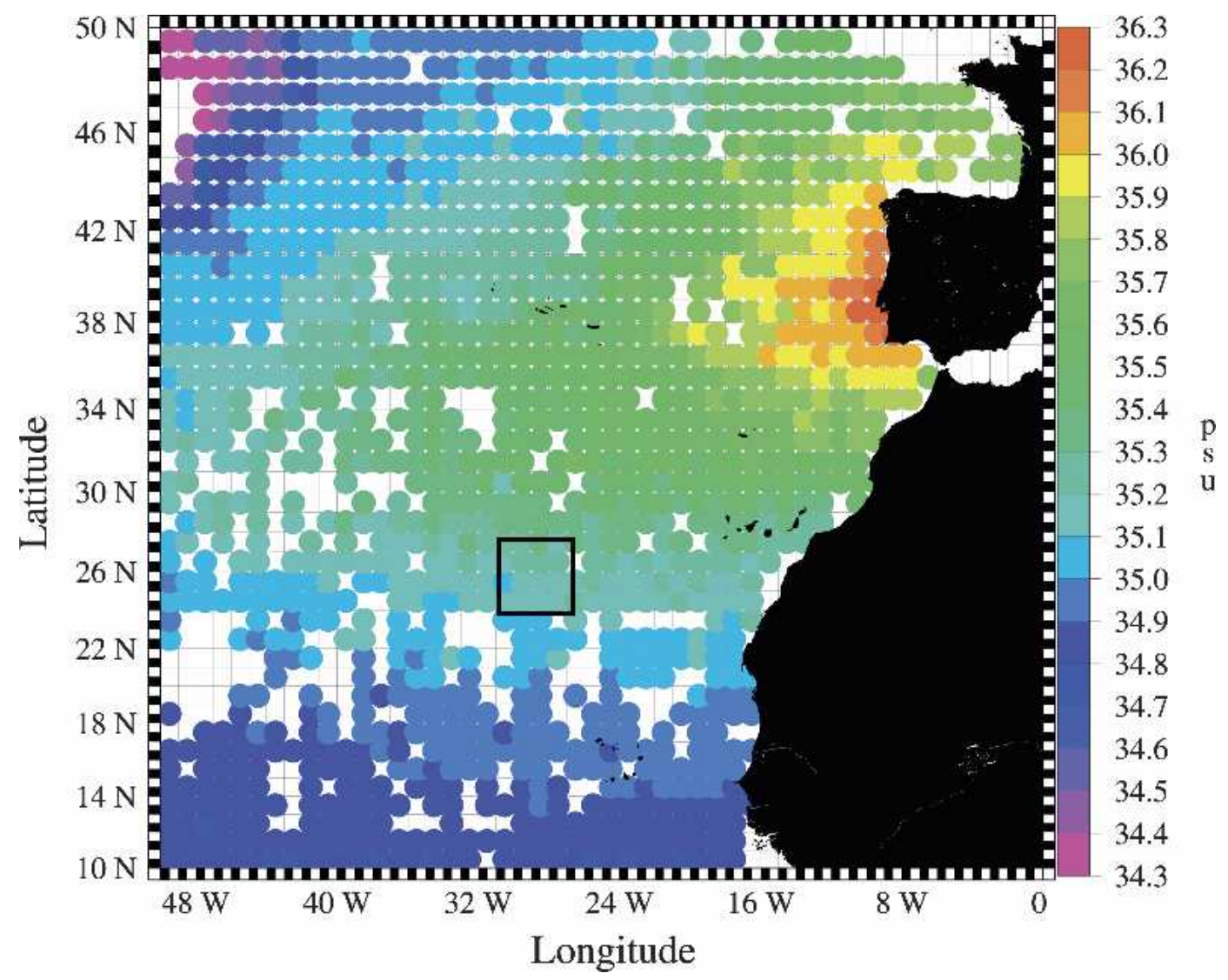

FIG. 1. A color collage of salinity on the $\gamma_{n}=27.6$ potential density surface (about 1000-m water depth). The broad region of high salinity represents the Mediterranean Salt Tongue. Fresher water to the northwest and south represent the influences of Labrador Sea Water and a highly modified version of Antarctic Intermediate Water, respectively. Data are from the Hydrobase climatological database (Curry 1996). The box centered at about $26^{\circ} \mathrm{N}$, $29^{\circ} \mathrm{W}$ encompasses the domain of high-resolution profiler stations obtained during the North Atlantic Tracer Release Experiment.

shear probes and fast-response temperature and conductivity sensors.

The HRP field program in NATRE was conducted during April 1992 southwest of the Canary Islands (Fig. 1). Sampling during this field program was organized in three grids of different resolution (Fig. 2):

- large: 100 profiles on a $400 \mathrm{~km} \times 400 \mathrm{~km}$ square grid with $25 \mathrm{n}$ mi station spacing, mostly to $2000-\mathrm{m}$ water depth;

- small: 16 profiles on a $25 \mathrm{~km} \times 25 \mathrm{~km}$ square grid with $5 \mathrm{n}$ mi station spacing, to 2000 -m water depth; and

- micro: 25 profiles on a $3.7 \mathrm{~km} \times 3.7 \mathrm{~km}$ L-shaped grid with $1 \mathrm{n}$ mi station spacing, mostly to $1200-\mathrm{m}$ water depth.

The NATRE HRP data are discussed in Polzin et al. (1995, 2003), Polzin (1996), St. Laurent and Schmitt (1999), and Polzin and Ferrari (2004).
The HRP survey in NATRE was near the center of a moored array deployed as part of the Subduction Experiment (R. A. Weller et al. 2004, unpublished manuscript). Mooring $\mathrm{C}$ at $25.5^{\circ} \mathrm{N}, 29^{\circ} \mathrm{W}$ was equipped with vector-averaging current meters at depths of 200, 300, 310,1500 , and $3500 \mathrm{~m}$. Two-year time series (summer 1991-spring 1993) of velocity and temperature are available at most depths.

\section{The temperature-salinity relation in NATRE}

The NACW of the main thermocline are characterized by an extremely tight $T-S$ relation and small thermohaline fluctuations (Fig. 3). Below, the water properties exhibit a distinct MW influence at the depths of $900-1400 \mathrm{~m}$ and the $T-S$ relation is much more variable. The $T-S$ variability disappears at even greater depths. The tightness of the NACW $T-S$ relation and the variability associated with MW has produced repeated commentary in the literature. 


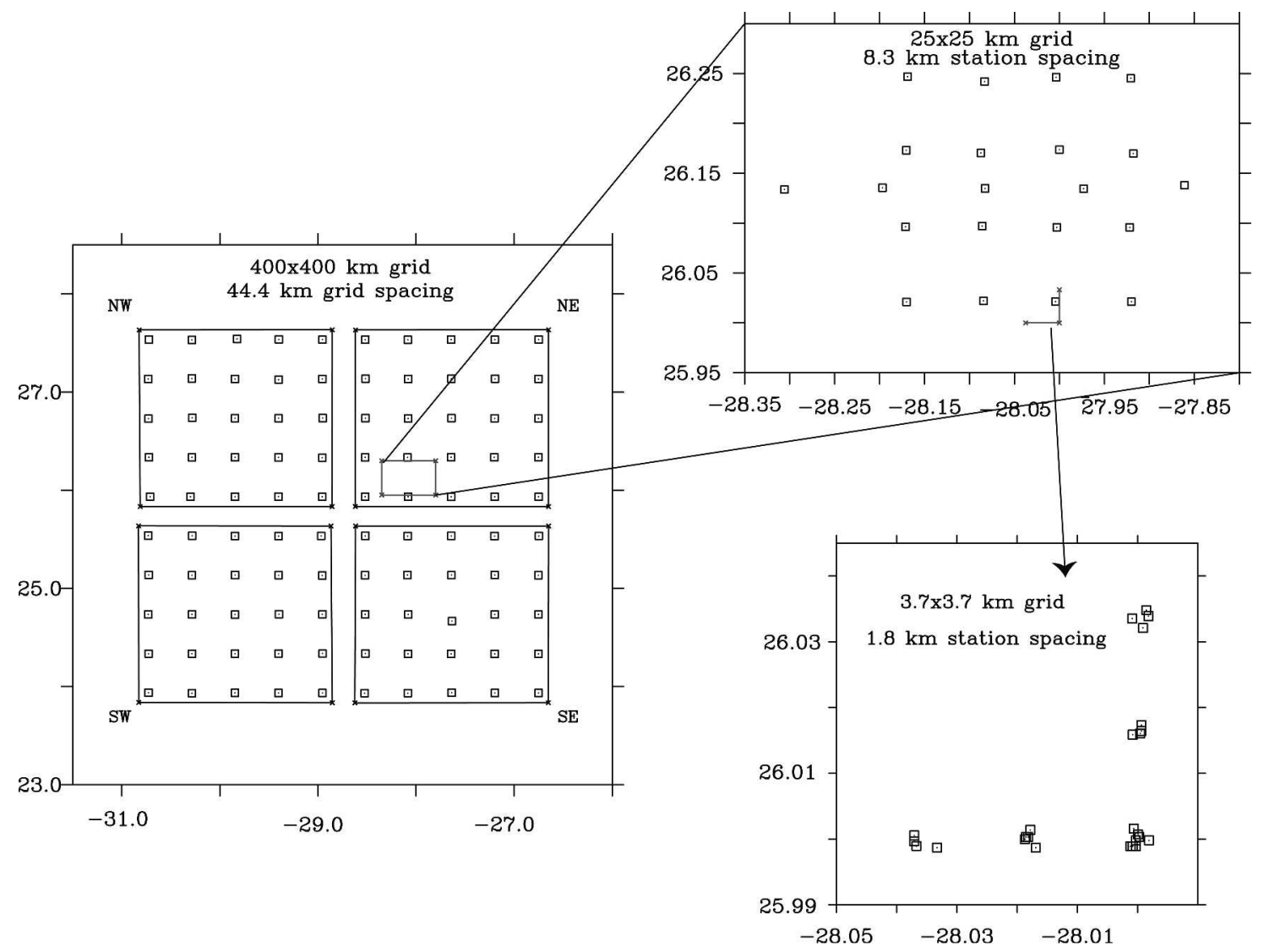

FIG. 2. Diagram of the three HRP grids used in this study. Square symbols denote HRP stations.

Because of the sampling in NATRE we are in position to comment about the scale of the $T-S$ variability. First, the $T-S$ variability is characterized by a lack of horizontal coherence: it is difficult to relate features in one $T-S$ profile with features in neighboring profiles 5 $\mathrm{n} \mathrm{mi}$ apart. Even at a $1 \mathrm{n} \mathrm{mi} \mathrm{grid} \mathrm{spacing,} \mathrm{the} \mathrm{lack} \mathrm{of}$ coherence is remarkable. This suggests that there is a rich finescale structure down to scales as small as one kilometer. Second, the $T-S$ variability is virtually independent of station spacing and grid size. In Fig. 3 we show $T-S$ profiles from stations in three different grids: a grid of $200 \mathrm{~km} \times 200 \mathrm{~km}$ in the center of the largescale grid with station spacings of $25 \mathrm{n} \mathrm{mi}$, the $25 \mathrm{~km} \times$ $25 \mathrm{~km}$ small-scale grid with station spacings of $5 \mathrm{n} \mathrm{mi}$, and the $3.7 \mathrm{~km} \times 3.7 \mathrm{~km}$ microscale grid with station spacings of $1 \mathrm{n} \mathrm{mi}$. The corresponding $T-S$ diagrams exhibit envelopes of about $0.15,0.15$, and 0.1 psu of salinity variations at temperatures of roughly $6^{\circ}-8^{\circ} \mathrm{C}$. This variability is nearly as large as the mean isopycnal salinity gradient of $0.2 \mathrm{psu}$ across the full $400 \mathrm{~km} \times 400$ $\mathrm{km}$ grid.

The richness of $T-S$ variability along isopycnals on a wide range of spatial scales is consistent with a scenario where water properties are stretched and folded by mesoscale eddies acting along isopycnals. We estimated temperature (and salinity) anomalies by binning data along neutral surfaces ${ }^{1}$ and computed the mean squared temperature (and salinity) differences for all available horizontal separations. These are the structure functions of temperature (and salinity) along neutral density surfaces (Fig. 4). Their dependence on spatial separation can be converted to the spectral domain and implies along-neutral density temperature (and salinity) spectra with a wavenumber roll-off of $k_{h}^{-1.2}$. This slope is close to the theoretical prediction of $k_{h}^{-1}$ associated with the inertial subrange for passive scalars stirred by a quasigeostrophic velocity field at scales smaller than the Rossby radius scale (Batchelor 1959). Moreover, if one is willing to assume that the salinity spectrum has a $k_{h}^{-1}$ slope between $200 \mathrm{~km}$ and $100 \mathrm{~m}$, that is, from the Rossby radius scale down to the scales

\footnotetext{
${ }^{1}$ Neutral surfaces are surfaces along which there is no buoyant restoring force (Jackett and McDougall 1997). With a suitable choice of reference pressure, neutral surfaces coincide with isopycnals.
} 
(a)

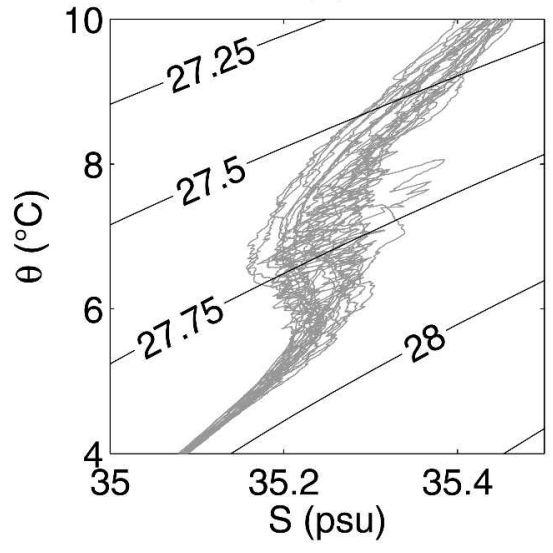

(b)

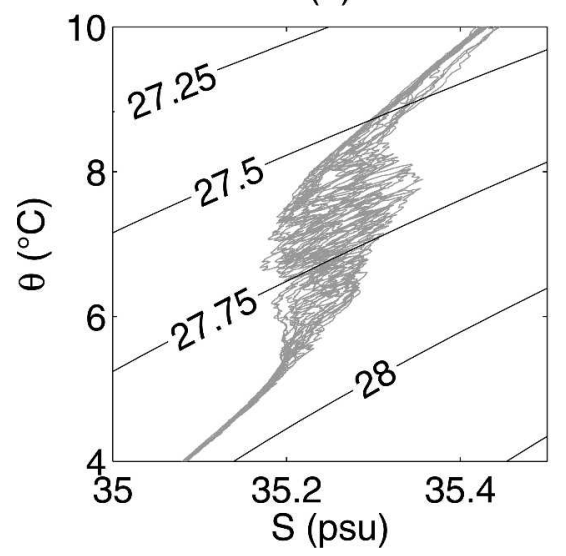

(c)

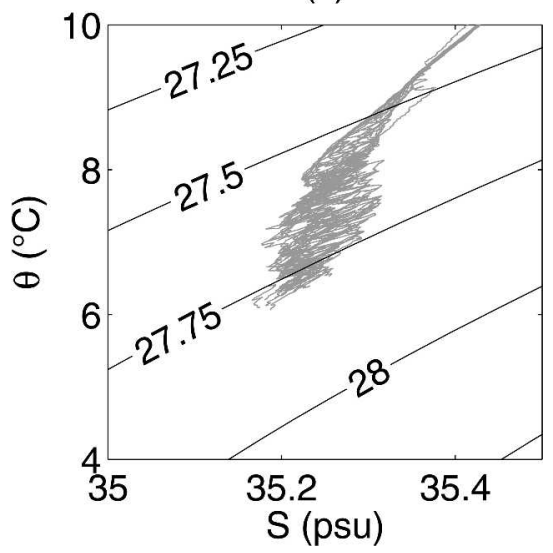

FIG. 3. The $T-S$ diagrams from the NATRE experiment from three different grids. (a) Profiles from 25 stations in a $200 \mathrm{~km} \times 200 \mathrm{~km}$ grid with $25 \mathrm{n}$ mi station spacing. These stations form the middle quadrant of the full grid. (b) Profiles from a $25 \mathrm{~km} \times 25 \mathrm{~km}$ grid at a $5 \mathrm{n} \mathrm{mi}$ station spacing. (c) Profiles from a microgrid of $3.7 \mathrm{~km} \times 3.7 \mathrm{~km}$ grid at a $1 \mathrm{n} \mathrm{mi}$ station spacing. Contours of constant neutral density are shown as black lines with a spacing of 0.25 $\mathrm{kg} \mathrm{m}^{-3} ; T-S$ variability is mostly along constant density lines at the MW level (neutral densities approximately between 27.5 and $27.8 \mathrm{~kg} \mathrm{~m}^{-3}$ ).

of turbulent patches generated by breaking internal waves, the ratio of variance between the small and large-scale grids is predicted to be be close to 1 and between the micro and large-scale grid close to 0.5 . These ratios are in remarkable agreement with the observed changes in isopycnal variability (Fig. 3).

The working hypothesis of this paper is that the large $T-S$ variability at the MW level is generated as follows. The outflow of Mediterranean Water creates a largescale salinity gradient along mean isopycnals. The horizontal strain and vertical shear associated with the mesoscale eddy field differentially advects $T-S$ properties and generates a rich finescale structure. Because mesoscale eddies are ineffective at creating small-scale density variability (MacVean and Woods 1980; Klein et al. 1997), the $T-S$ variability is near perfectly compensat- ing in density; that is, $T$ anomalies are largely offset by $S$ anomalies with equal and opposite effect on density. The theoretical explanation is as follows (Klein et al. 1997). In stratified rotating turbulence density variance and kinetic energy are transferred to large horizontal and vertical scales through an inverse energy cascade. Thus density and velocity profiles tend to develop a low vertical mode structure. Temperature-salinity variability along density surfaces is instead transferred to small scales because it is dynamically passive and not affected by the inverse cascade. Thus one expects sharp compensated $T-S$ gradients and smooth density profiles. The $T-S$ variability observed in the NATRE dataset is not apparent in the profiles of potential density as increased $N^{2}$ variability. Polzin et al. (2003) investigated the fraction of buoyancy frequency variability $N^{2}$, 


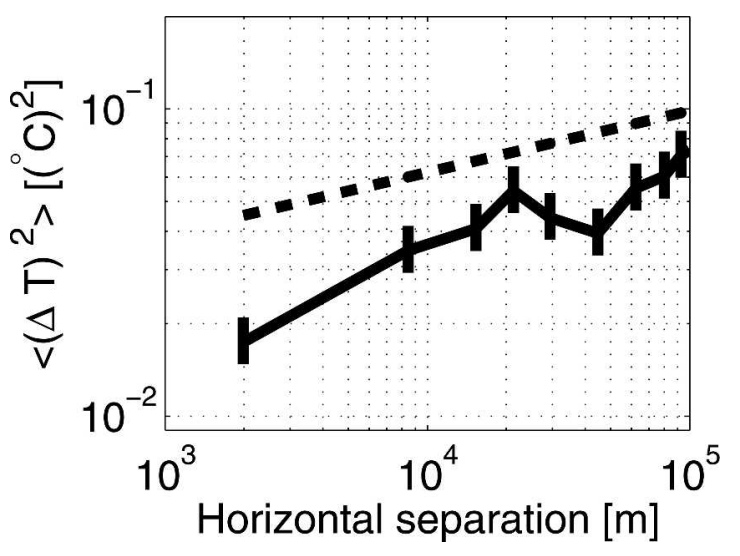

FIG. 4. Structure function of potential temperature fluctuations along neutral density surfaces at the Mediterranean Water level, i.e., neutral densities between 27.5 and $27.7 \mathrm{~kg} \mathrm{~m}^{-3}$. The structure function is defined as $\left\langle(\Delta \theta)^{2}\right\rangle=\left\langle[\theta(x+r)-\theta(x)]^{2}\right\rangle$, where the average is carried over all measurements a distance $|r|$ apart, along the same density surface. In logarithmic scale $\left\langle(\Delta \theta)^{2}\right\rangle$ grows linearly with separation $|r|$ : a linear fit gives a slope of approximately 0.2 represented as the black dashed line. This result can be converted in spectral space and corresponds to a spectrum with a roll-off of $k_{h}^{-1.2}$ for scales between 100 and $2 \mathrm{~km}$. Spectral slopes of temperature fluctuations along density surfaces in the Central Waters are similar, but the spectral levels are much smaller.

which could be assigned to mesoscale and submesoscale balanced motions, as opposed to isopycnal straining associated with internal waves. The same technique can be applied at the Mediterranean Water level, and one finds that $N^{2}$ variability associated with balanced motions is much smaller than that in temperature and salinity.

In the absence of other competing processes, the creation of ever-sharper gradients proceeds without bound. Only at scales of a few hundred meters in the horizontal and some tens of meters in the vertical do three-dimensional turbulent processes arrest the $T-S$ filamentation. This is consistent with the lack of horizontal coherence in $T-S$ profiles a few kilometers apart.

The cascade of $T-S$ variance to small scales follows a different path in the NACW. At this level the mean isopycnal thermohaline gradient is weak and eddy stirring is inefficient at generating $T-S$ variability. As a result the $T-S$ relation is tight. In the next few sections we discuss an approach to assess quantitatively the role of eddy stirring and diapycnal mixing in microstructure data and we apply it to the NATRE observations.

\section{Finescale and microscale variability in the ocean}

The goal of this paper is to identify what processes generate the small-scale $T-S$ fluctuations in the NATRE region. Following a "Reynolds decomposition" of variables into a slowly changing mean and fluc- tuations, we ask what physics generates the thermohaline fluctuations. This variability can be quantified in terms of the variance $\overline{C^{\prime 2}}$, where $C^{\prime}$ represents fluctuation of a tracer, be it $T$ or $S$. The variance satisfies a conservation equation of the form

$$
\begin{aligned}
\frac{\partial \overline{C^{\prime 2}}}{\partial t}+\nabla \cdot\left(\overline{\mathbf{u}} \overline{C^{\prime 2}}\right. & \left.+\overline{\mathbf{u}^{\prime} C^{\prime 2}}-\kappa_{C} \nabla \overline{C^{\prime 2}}\right)+2 \overline{\mathbf{u}^{\prime} C^{\prime}} \cdot \nabla \bar{C} \\
& =-2 \kappa_{C} \overline{\nabla C^{\prime} \cdot \nabla C^{\prime}} .
\end{aligned}
$$

The terms on the lhs represent both the generation and transport of tracer fluctuations, while the term on the rhs is the dissipation of tracer variance by molecular motions, with $\kappa_{C}$ the molecular diffusivity of $C$. If the fluctuations are statistically stationary and homogeneous, then the time-dependent and divergence terms on the left-hand side of (4.1) vanish and there is a balance between variance production by eddy fluxes acting on the mean gradient and molecular dissipation,

$$
\overline{\mathbf{u}^{\prime} C^{\prime}} \cdot \nabla \bar{C}=-\kappa_{C} \overline{\nabla C^{\prime} \cdot \nabla C^{\prime}} \text {. }
$$

This is the Osborn-Cox formula, if $C$ is temperature (Osborn and Cox 1972). The lhs represents the generation of variance by eddies stirring the large-scale gradients. The rhs is the removal of variance by molecular mixing. A considerable literature exists on how to infer the eddy fluxes of temperature from direct measures of tracer dissipation using fast response thermistors.

The Reynolds decomposition assumes spectral gaps between mean and fluctuations. Operationally, the spectral gap is invoked to neglect correlations between mean and fluctuations, $\overline{\mathbf{u}^{\prime} \bar{C}}=0$, and to turn triple correlations into a fluctuation flux times a mean gradient, $\overline{\mathbf{u}^{\prime} C^{\prime} \cdot \nabla \bar{C}}=\overline{\mathbf{u}^{\prime} C^{\prime}} \cdot \nabla \bar{C}$. To assess the spectral gap assumption, it is essential to define which processes are included in the mean and which is left in the fluctuations. Osborn and Cox used a scale analysis in which fluctuations were separated from the mean according to spatial scale. Fluctuations were ostensibly the microscale component that supports across-isopycnal transport. This requires the mean to include internal waves and mesoscale eddies. The original analysis was somewhat inconsistent with this, interpreting the mean velocity and temperature as representative of the largescale circulation only. This inconsistency is crucial because it neglects scale-transformation processes linking dissipation scales to energetic intermediate scales.

\section{a. The triple decomposition}

To examine the role of scale-transforming processes, we consider a triple decomposition of variables into mean, mesoscale fluctuations, and microscale turbulence (Joyce 1977; Davis 1994; Garrett 2001). To mi- 
croscale turbulence we assign all scales between statically unstable overturns and those that support direct molecular dissipation, that is, scales between a few meters and a few millimeters. This category includes shear instabilities, convection, and double diffusion. The mesoscale fluctuations consist of mesoscale eddies, internal waves, and vortical modes, that is, scales between a hundred kilometers and a few meters. The mean represents the large-scale water mass properties and the ocean general circulation.

To proceed with the triple Reynolds decomposition, we must assume that there is a spectral gap between the mean and mesoscale fluctuations and another gap between mesoscale fluctuations and microscale turbulence. It is not clear whether such gaps exist (Davis 1994). Mesoscale eddies overlap with the meanders of the large-scale circulation. Internal waves (Polzin et al. 2003) can have scales as large as mesoscale eddies and as short as microscale turbulence. Despite this lack of spectral gaps, the triple decomposition remains useful in discussing scale transformations of $T-S$ variance from the large-scale down to the dissipative scales. It so happens that mesoscale eddy fluxes dominate the cascade from the large to the finescale, and turbulence dominates the cascade from the fine- to the microscale in the NATRE dataset. Certainly a gap exists between balanced eddies and unbalanced turbulent motions, and thus we believe the issue of scale separation is not too problematic for the problem at hand.

Let us write $C=C_{m}+C_{e}+C_{t}$, with subscripts $m, e$, and $t$ denoting mean, mesoscale fluctuations, and turbulence, respectively. For any quantity $C$, let us also write $\tilde{C}$ for the average of $C$ over a spatial scale large in comparison with the scale of turbulence, but small in comparison with that of the mesoscale fluctuations, and $\langle C\rangle$ for the average of $C$ over a spatial scale long in comparison with that of the mesoscale fluctuations, but short in comparison with that of the mean state. Substituting the three-component decomposition for the tracer $C$ and the momentum $\mathbf{u}$ into the tracer budget we obtain

$$
\begin{aligned}
\partial_{t} C_{m}+\mathbf{u}_{m} \cdot \nabla C_{m}-\kappa_{C} \nabla^{2} C_{m}= & -\boldsymbol{\nabla} \cdot\left\langle\mathbf{u}_{e} C_{e}\right\rangle \\
& -\boldsymbol{\nabla} \cdot\left\langle\mathbf{u}_{t} C_{t}\right\rangle .
\end{aligned}
$$

The first term on the rhs of (4.3) represents tracer fluxes driven by mesoscale fluctuations. The second term is due to microscale turbulent fluxes.

The corresponding variance budgets of mesoscale and microscale fluctuations are

$\frac{1}{2}\left(\partial_{t}+\mathbf{u}_{m} \cdot \boldsymbol{\nabla}\right)\left\langle C_{e}^{2}\right\rangle+\nabla \cdot\left(\frac{1}{2}\left\langle\mathbf{u}_{e} C_{e}^{2}\right\rangle+\left\langle\widetilde{\mathbf{u}_{t} C_{t}} C_{e}\right\rangle-\kappa_{C}\left\langle C_{e} \boldsymbol{\nabla} C_{e}\right\rangle\right)=-\left\langle\mathbf{u}_{e} C_{e}\right\rangle \cdot \nabla C_{m}+\left\langle\widetilde{\mathbf{u}_{t} C_{t}} \cdot \nabla C_{e}\right\rangle-\kappa_{C}\left\langle\left|\nabla C_{e}\right|^{2}\right\rangle$,

and

$\frac{1}{2}\left(\partial_{t}+\mathbf{u}_{m} \cdot \boldsymbol{\nabla}\right)\left\langle C_{t}^{2}\right\rangle+\nabla \cdot\left(\frac{1}{2}\left\langle\mathbf{u}_{t} C_{t}^{2}\right\rangle+\frac{1}{2}\left\langle\mathbf{u}_{e} \widetilde{C_{t}^{2}}\right\rangle-\kappa_{C}\left\langle C_{t} \boldsymbol{\nabla} C_{t}\right\rangle\right)=-\left\langle\widetilde{\mathbf{u}_{t} C_{t}} \cdot \nabla C_{e}\right\rangle-\left\langle\mathbf{u}_{t} C_{t}\right\rangle \cdot \nabla C_{m}-\kappa_{C}\left\langle\left|\nabla C_{t}\right|^{2}\right\rangle$

These budgets relate eddy fluxes to measurable quantities like variance dissipation. Simplified versions of these equations will be used for the analysis of the NATRE observations.

\section{b. Temperature variance budget}

Let us apply the triple-scale variance budget analysis to potential temperature $\theta$. Assuming stationarity, so that variances do not change with time, and homogeneity, so that divergence terms may be ignored, the variance budgets for the mesoscale fluctuations and microscale turbulence reduce to (Garrett 2001)

$$
\begin{aligned}
& \underbrace{\left\langle\mathbf{u}_{e} \theta_{e}\right\rangle \cdot \boldsymbol{\nabla} \theta_{m}}_{[1]}-\underbrace{\left\langle\widetilde{\mathbf{u}_{t} \theta_{t}} \cdot \boldsymbol{\nabla} \theta_{e}\right\rangle}_{[2]}=0, \\
& \underbrace{\left\langle\mathbf{u}_{t} \theta_{t}\right\rangle \cdot \boldsymbol{\nabla} \theta_{m}}_{[3]}+\underbrace{\left.\widetilde{\mathbf{u}_{t} \theta_{t}} \cdot \boldsymbol{\nabla} \theta_{e}\right\rangle}_{[2]}=-\underbrace{\frac{1}{2}\langle\chi\rangle}_{[4]}, \text { and }
\end{aligned}
$$

$$
\underbrace{\left\langle\mathbf{u}_{t} \theta_{t}\right\rangle \cdot \boldsymbol{\nabla} \theta_{m}}_{[3]}+\underbrace{\left\langle\mathbf{u}_{e} \theta_{e}\right\rangle \cdot \nabla_{n} \theta_{m}}_{[1]}=-\underbrace{\frac{1}{2}\langle\chi\rangle}_{[4]},
$$

where $\chi \equiv \kappa_{\theta}\left|\nabla \theta_{t}\right|^{2}$ is the instantaneous rate of temperature dissipation and $\kappa_{\theta}$ is the molecular diffusivity. The last equation is simply the sum of the first two. Davis (1994) carefully checked the relative size of the divergence and accumulation terms that we dropped in writing (4.6) through (4.8). His conclusion was that advection and accumulation of temperature variance can be neglected in regions without strong upwelling, as is the case in NATRE. However the triple products; that is, the divergence of the turbulent and eddy fluxes of variance-represent exchanges of variance between the various scales of motion and cannot be dismissed by scale analysis. Here we assume, as a hypothesis, that the divergence of the triple products can be neglected. This hypothesis will be checked by testing whether the balances in (4.6) through (4.8) are satisfied in the observations. 
Equation (4.6) states that variance generated by mesoscale eddies acting on the mean state [1] is passed on to the microscale by turbulence acting on the mesoscale fluctuations [2]. The next equation shows that the dissipation of thermal variance [4] may be regarded as coming from the turbulence acting on both the mean state [3] and the fluctuations [2]. This is the OsbornCox model with additional terms that represent the processes linking dissipation to the mesoscale fluctuations.

The variance budgets at the meso- and microscales are nicely summarized with a diagram proposed by Garrett (2001):

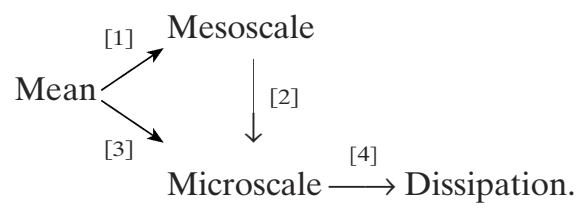

Equation (4.6) represents the upper branch of the path of variance: mesoscale eddies extract variance from the mean [1] and transfer it to microscale [2]. Equation (4.7) is, instead, a description of the lower branch: turbulent motions extract variance from the mean [3] and the mesoscale [2] and deliver it to the microscale [4] where it is dissipated. Notice that the direction of the arrows is a matter of sign conventions and does not imply a one-way transfer of variance.

The variance production by mesoscale motions stirring the mean temperature distributions [1] is dominated by geostrophically balanced eddies. Polzin and Ferrari (2004) and Ferrari and Lvov (2004, unpublished manuscript) show that the flux associated with vortical modes and internal waves is several orders of magnitude smaller than that produced by balanced eddies. ${ }^{2}$ Observational (Ledwell et al. 1993) and numerical (Gent and McWilliams 1990) studies suggest that eddies transport temperature mostly along neutral surfaces with a negligible diapycnal component. Thus $\left\langle\mathbf{u}_{e} \theta_{e}\right\rangle \cdot \boldsymbol{\nabla} \theta_{m} \approx\left\langle\mathbf{u}_{e} \theta_{e}\right\rangle \cdot \nabla_{n} \theta_{m}$, where $\boldsymbol{\nabla}_{n} \theta_{m}$ is the gradient along the neutral density surface.

Turbulent fluxes, [2] and [3], are the focus of traditional microscale studies (Osborn and Cox 1972; Gregg 1989). These fluxes are directed both along and across isopycnals, but the isopycnal projection is typically very small and can be neglected: turbulent fluxes represent

\footnotetext{
${ }^{2}$ Internal waves become an important transport mechanism at meter scales where nonlinear effects are large and overturns and breaking occur. According to our definition of averages, internal wave breaking in included in the microstructure turbulence.
}

diapycnal mixing of the tracer. Turbulent processes include shear, convective, and doubly diffusive instabilities. Shear instabilities trigger wave breaking and drive variance from the mean and mesoscales to the dissipation scales: the corresponding path of variance follows the arrows [2] and [3]. The same is true for convective instabilities. Double diffusive instabilities, driven by the different molecular diffusivities of heat and salt, can move variance both upscale and downscale. Thus the path of variance represented by [2] and [3] can have the arrows going either way. Upscale transfer of variance corresponds to the generation of large-scale temperature fluctuations using energy released by small-scale double-diffusive instabilities. The $T-S$ staircases found in the Eastern North Atlantic grow to vertical scales of tens of meters and are coherent in the horizontal over hundreds of kilometers. They are believed to be an example of an upscale doubly diffusive variance transfer.

Equation (4.8) is at the heart of our analysis. The temperature variance that is dissipated at molecular scales [4] can be either generated by eddy stirring along isopycnals [1] or by diapycnal mixing across isopycnals [3]. The first process generates compensated $T-S$ variability, while the second process tends to tighten the $T-S$ relationship by combining water types.

\section{c. Turbulent kinetic energy budget and buoyancy fluxes}

Microscale temperature variance can be produced by both isopycnal stirring (mesoscale eddies) and diapycnal mixing (turbulence). Microscale buoyancy ${ }^{3}$ variance, instead, is mostly produced by diapycnal mixing: mesoscale motions are directed along neutral surfaces and are not very efficient at folding buoyancy contours. It thus appears that an analysis of the buoyancy budget might shed light on the turbulent fluxes in isolation of mesoscale motions. However the buoyancy variance budget is not used in practice because of the difficulty of measuring the haline component of buoyancy dissipation. ${ }^{4}$

Osborn (1980) pointed out that turbulent buoyancy fluxes can be related to eddy kinetic energy dissipation, which can be accurately measured with airfoil shear probes. The method is based on the kinetic energy budget. By applying the triple decomposition to the mo-

\footnotetext{
${ }^{3}$ Buoyancy is defined as $b=-g \rho / \rho_{0}$, where $g$ is the acceleration of gravity, $\rho$ the density of seawater, and $\rho_{0}$ a reference constant density.

${ }^{4}$ It is only very recently that Nash and Moum (2002) reported estimates of haline dissipation.
} 
mentum equations, we can derive budgets for the turbulent kinetic energy at the microscale. Under the usual assumptions of statistical homogeneity and isotropy, the microscale eddy kinetic energy budget takes the form

$$
\left\langle\widetilde{u_{t i} u_{t j}} \frac{\partial u_{e i}}{\partial x_{j}}\right\rangle+\left\langle u_{t i} u_{t j}\right\rangle \frac{\partial u_{m i}}{\partial x_{j}}=\left\langle w_{t} b_{t}\right\rangle-\langle\epsilon\rangle,
$$

where the subscripts $(i, j)$ indicate the three Cartesian directions, and repeated subscripts denote summation. The rate of turbulent kinetic energy dissipation $\langle\epsilon\rangle$ is given by

$$
\langle\epsilon\rangle=\left\langle\nu \frac{\partial u_{t i}}{\partial x_{j}}\left(\frac{\partial u_{t i}}{\partial x_{j}}+\frac{\partial u_{t j}}{\partial x_{i}}\right)\right\rangle,
$$

with $\nu$ the molecular viscosity. The budget in (4.10) represents a balance between shear production of turbulent kinetic energy (two terms on the lhs), conversion of kinetic energy into potential energy (first term on the rhs), and dissipation (second term on the lhs). Shear production is by turbulent eddy stresses, $\overline{u_{t i} u_{t j}}$, acting on the low frequency shear of the mean circulation, $\partial u_{m i} l$ $\partial x_{j}$, and on the higher frequency shear of mesoscale motions, $\partial u_{e i} / \partial x_{j}$, be it coherent eddies or internal waves. The fraction of turbulent energy production used to raise potential energy is quantified through the flux Richardson number $R_{f}$,

$$
R_{f}=\frac{\left\langle w_{t} b_{t}\right\rangle}{\left\langle\widetilde{u_{t i} u_{t j}} \partial u_{e i} / \partial x_{j}\right\rangle+\left\langle u_{t i} u_{t j}\right\rangle \partial u_{m i} / \partial x_{j}} .
$$

In terms of the flux Richardson number, the turbulent kinetic energy budget takes the form

$$
\left\langle w_{t} b_{t}\right\rangle=-\Gamma_{t}\langle\epsilon\rangle
$$

where the proportionality factor $\Gamma_{t} \equiv R_{f} /\left(1-R_{f}\right)$ is the "turbulent mixing efficiency" (Ruddick et al. 1997).

There is an extensive literature on how to estimate the turbulent mixing efficiency in stably stratified fluids. The discussion is typically framed in terms of the flux Richardson number $R_{f}$ and the density ratio $R_{\rho}=$ $\alpha\left\langle\partial_{z} \theta\right\rangle / \beta\left\langle\partial_{z} S\right\rangle$, where $\alpha$ and $\beta$ are the coefficients of thermal expansion and haline contraction. For shear instability, laboratory experiments (Linden 1979; Rohr et al. 1984) suggest $0.05 \leq R_{f} \leq 0.25$. In oceanic applications shear instabilities are associated with breaking internal waves and $R_{f}$ is found to be close to its maximum value 0.25 (Polzin 1996). Thus $\Gamma_{t}=0.2$ is typically used to estimate buoyancy fluxes from (4.13) (Moum 1996; St. Laurent and Schmitt 1999). For weak shear, production ceases and buoyancy fluxes can result from small-scale instabilities driven by the difference between the molecular diffusivities of heat and salt. In regions with stable temperature stratification and unstable salinity stratification, the instability is in the form of "salt fingers," that is, $O(1 \mathrm{~cm})$ plumes that transport salt more efficiently than heat and tend to restratify the water column. Salt fingers appear to dominate the turbulent production for $1<R_{\rho}<2$ and $\mathrm{Ri}>1$, and have $\Gamma_{t}>$ 0.2 (St. Laurent and Schmitt 1999).

\section{Turbulent diffusivity estimates}

Turbulent fluxes of buoyancy can be related to buoyancy stratification by a flux-gradient relationship with a diapycnal turbulent diffusivity $K_{\rho}$,

$$
\left\langle w_{t} b_{t}\right\rangle=-K_{\rho} \partial_{z} b_{m} .
$$

Combining this expression for $\left\langle w_{t} b_{t}\right\rangle$ with the formula in (4.13), $K_{\rho}$ can be expressed in terms of quantities available from observations, $\langle\epsilon\rangle$ and $\partial_{z} b_{m}$, and the turbulent mixing efficiency,

$$
K_{\rho}=\Gamma_{t} \frac{\langle\epsilon\rangle}{\partial_{z} b_{m}} .
$$

We refer to $K_{\rho}$ as a diapycnal turbulent diffusivity because (5.1) is the oceanic approximation of the fluxgradient relationship, $\left\langle\mathbf{u}_{t} b_{t}\right\rangle=-K_{\rho} \nabla b_{m}$, where the mean buoyancy gradient is dominated by the vertical component.

To derive estimates of turbulent diffusivity, we employ all vertical profiles from the $400 \mathrm{~km} \times 400 \mathrm{~km}$ square grid in Fig. 2. Each profile includes a full record of dissipation data down to $2000 \mathrm{~m}$ at a spacing of $0.5 \mathrm{~m}$ and can be used to estimate the eddy kinetic energy balance in (4.13). The large-scale average operator \langle\rangle represents (i) a horizontal average over the survey lateral scale, (ii) a vertical average over $O(100) \mathrm{m}$, and (iii) a time average over the 18-day survey.

The mean fields are derived by averaging all variables along neutral-density surfaces $\gamma_{n}$. Uncertainty in the mean field estimates are attributed to mesoscale and microscale motions (instrumental noise is significantly smaller than the observed fluctuations for all variables). Mean vertical gradients $\partial_{z} \theta_{m}, \partial_{z} S_{m}$, and $\partial_{z} b_{m}$, are calculated from $O(100)$-m linear fits to $\theta_{m}\left(z_{n}\right)$, $S_{m}\left(z_{n}\right)$, and $b_{m}\left(z_{n}\right)$, where $z_{n}$ is the mean depth of each surface $\gamma_{n}$. Similar choices were made by St. Laurent and Schmitt (1999) in their analysis of the NATRE microstructure data from the upper $800 \mathrm{~m}$.

Estimates of $\langle\epsilon\rangle$ and its uncertainty are obtained with standard procedures given in the appendix. Assuming conservatively that dissipation is associated to shear 


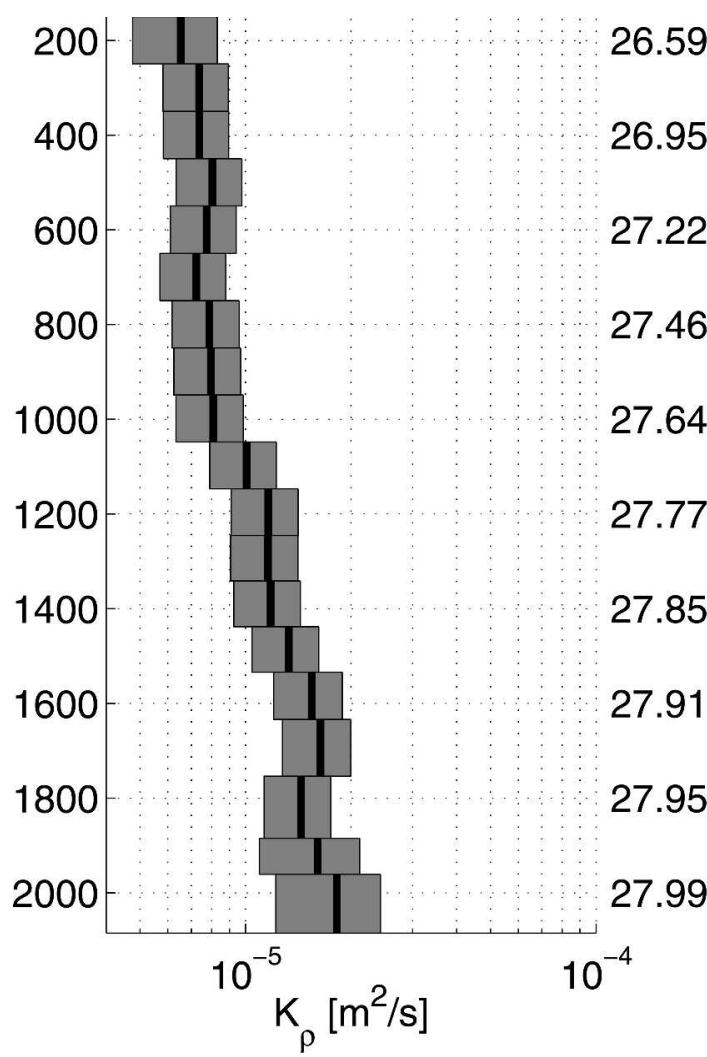

FIG. 5. Estimates of the turbulent diffusivity $K_{\rho}=0.2\langle\varepsilon\rangle / \partial_{z} b_{m}$ for the NATRE region. For each neutral density layer, mean values are shown as a vertical line, and the uncertainty as a shaded gray box. The vertical axis is restricted to neutral density layers that did not outcrop within the survey region. The near-surface layers have much larger fluxes because of the enhanced turbulence generated by air-sea interactions and are not the focus of this paper.

turbulence, we set $\Gamma_{t}=0.2$ in (5.2) and estimate $K_{\rho}$. Results of the calculation are shown in Fig. 5. The turbulent diffusivity is nearly constant in the upper $1000 \mathrm{~m}$, with values close to $0.7 \times 10^{-5} \mathrm{~m}^{2} \mathrm{~s}^{-1}$, and grows to 1.5 $\times 10^{-5} \mathrm{~m}^{2} \mathrm{~s}^{-1}$ throughout the density layers characterized by MW. This range of values is typical of diapycnal diffusivities measured in the open ocean away from boundaries.

St. Laurent and Schmitt (1999) find that in the upper $400 \mathrm{~m}$ of the water column turbulent fluxes are characterized by both fingering convection and shear dispersion. Their analysis suggests that $0.2<\Gamma_{t}<3$. By setting $\Gamma_{t}=0.2$ we underestimate $K_{\rho}$. In the next section we show that ignoring double diffusive effects is justified below $400 \mathrm{~m}$. Even above, the bias in $K_{\rho}$ is less than $40 \%$ and does not affect any of our conclusions.

Ledwell et al. (1993) estimated the NATRE turbulent diffusivity, at $300 \mathrm{~m}$, to be $0.11 \pm 0.02 \mathrm{~m}^{2} \mathrm{~s}^{-1}$, a value somewhat larger than what is reported here.
However, Ledwell and collaborators used tracer release techniques to obtain their estimate, instead of microstructure dissipation measurements. Thus their turbulent diffusivity is not the diffusivity of buoyancy, but the diffusivity of a passive tracer. St. Laurent and Schmitt (1999) find that double diffusion is active in the upper thermocline at $300 \mathrm{~m}$ and can account for this difference. Fingers transport salt and passive tracers more effectively than buoyancy and enhance their diffusivities. Fingers can also modify buoyancy transports, but this effect is very weak at the NATRE site. Our estimate of $K_{\rho}$ should be regarded as the turbulent diffusivity generated by shear turbulence.

\section{Temperature variance analysis}

In section $4 \mathrm{~b}$ we derived the microstructure variance budget,

$$
\underbrace{\left\langle\mathbf{u}_{t} \theta_{t}\right\rangle \cdot \boldsymbol{\nabla} \theta_{m}}_{[3]}+\underbrace{\left\langle\mathbf{u}_{e} \theta_{e}\right\rangle \cdot \nabla_{n} \theta_{m}}_{[1]}=-\underbrace{\frac{1}{2}\langle\chi\rangle}_{[4]} .
$$

Microstructure measurements provide a direct estimate of $\chi$. In the following we discuss how to infer the two terms on the lhs of (6.1), that is, the production of microstructure variance by microstructure turbulence and by mesoscale fluctuations.

\section{a. Variance production by mesoscale fluctuations}

The production of microstructure variance by mesoscale stirring, [1] in (6.1), is dominated by balanced eddies with smaller contributions from vortical modes and internal waves (Polzin and Ferrari 2004; Ferrari and Lvov 2004, unpublished manuscript). We attempted to estimate the mesoscale eddy fluxes $\left\langle\mathbf{u}_{e} \theta_{e}\right\rangle$ by computing the correlations between $\mathbf{u}_{e}$ and $\theta_{e}$ from current-meter data. However, the 2-yr time series were too short and failed to produce a significant correlation between the subinertial temperature and velocity fluctuations.

Alternatively, we assume a flux-gradient representation,

$$
\left\langle\mathbf{u}_{e} \theta_{e}\right\rangle \cdot \nabla_{n} \theta_{m}=-K_{e}\left|\nabla_{n} \theta_{m}\right|^{2},
$$

and reduce the problem to one of estimating the mesoscale effective diffusivity $K_{e}$. Notice that eddies stir along neutral surfaces so that $K_{e}$ is an along-neutralsurface diffusivity. This is the reason why only the along-neutral-surface gradient $\boldsymbol{\nabla}_{n} \theta_{m}$ appears in (6.2). By definition $\nabla_{n} \theta_{m}=\left(\partial_{x} \theta_{m}, \partial_{y} \theta_{m}\right)$ with $x$ and $y$ coordinates along a neutral surface. 
TABLE 1. Mesoscale effective diffusivities in the NATRE region as estimated from previous studies. For studies that report both a zonal and a meridional effective diffusivity, we computed the projection in the direction of the large-scale temperature gradient. Only the along-gradient component generates temperature variance, which is the variable of interest in this study. In the NATRE region the large-scale temperature gradient is meridional at the Mediterranean Water level and close to zero elsewhere.

\begin{tabular}{lclrr}
\hline \multicolumn{1}{c}{ Source } & \multicolumn{1}{c}{ Region } & \multicolumn{1}{c}{ Data } & Depth $(\mathrm{m})$ & $K_{e}\left(\mathrm{~m}^{2} \mathrm{~s}^{-1}\right)$ \\
\hline Joyce et al. (1998) & $19^{\circ}-34^{\circ} \mathrm{N}$ & Floats & 250 & 1100 \\
Ledwell et al. (1998) & $26^{\circ} \mathrm{N}, 29^{\circ} \mathrm{W}$ & Tracer release & 300 & 1000 \\
Jenkins (1998) & $18^{\circ}-33^{\circ} \mathrm{N}$ & He & 500 & 850 \\
Armi and Stommel (1983) & $22^{\circ}-31^{\circ} \mathrm{N}$ & Hydrography & 800 & 500 \\
Spall et al. (1993) & $20^{\circ}-28^{\circ} \mathrm{N}$ & Floats & 1100 & 350 \\
\hline
\end{tabular}

There have been a number of independent estimates of $K_{e}$ in the NATRE region (Table 1). Values are close to $1000 \mathrm{~m}^{2} \mathrm{~s}^{-1}$ in the upper $800 \mathrm{~m}$ of the water column and drop to $350-500 \mathrm{~m}^{2} \mathrm{~s}^{-1}$ at the MW level. For studies that report both a meridional and a zonal diffusivity, we consider the projection of the diffusivity along the mean temperature gradient. However, in all studies, the zonal-meridional anisotropy is within the error bars and thus statistically insignificant.

The decrease of $K_{e}$ with depth is evident in the composite diagram in Fig. 6, but the exact vertical structure of $K_{e}$ cannot be inferred with such a sparse vertical sampling. The gaps could be filled by using one of many parameterization schemes that relate the mesoscale effective diffusivity to mean properties of the flow (Green 1970; Stone 1972; Larichev and Held 1995; Pavan and Held 1996; Visbeck et al. 1997). Here we prefer to rely on observations and attempt a direct estimation of $K_{e}$ from a suite of mooring and CTD data.

We estimate $K_{e}$ using "mixing length" theory (Armi and Stommel 1983). According to mixing length theory, the diffusivity is related to the characteristic velocity and length scale of the transfer process,

$$
K_{e} \approx c_{e} U_{e} L_{e}
$$

where $U_{e}$ is the rms turbulent velocity along neutral surfaces, $L_{e}$ is a measure of the lateral transfer scale, and $c_{e}$ is an efficiency factor.

The characteristic eddy velocity $U_{e}$ is estimated as the subinertial rms eddy velocity from the mooring data. Rms velocities decrease from $3 \mathrm{~cm} \mathrm{~s}^{-1}$ at $200 \mathrm{~m}$ down to $2 \mathrm{~cm} \mathrm{~s}^{-1}$ at $1500 \mathrm{~m}$. Measurements are available only at depths of 200, 300, 310, 1500, and $3500 \mathrm{~m}$. To obtain values at intermediate depths we simply interpolated the data. Wunsch (1997) shows that most of the oceanic eddy kinetic energy is concentrated in the barotropic and first two baroclinic modes. Thus the results will not be overly sensitive to the lack of vertical resolution.

The lateral eddy scale $L_{e}$ is the length for which an eddy carries the value of a conservative tracer, before it is mixed with its surroundings. Using mixing length arguments, we estimate $L_{e}$ as (Armi and Stommel 1983)

$$
L_{e}=\theta_{\mathrm{rms}} /\left|\nabla_{n} \theta_{m}\right|
$$

where $\theta_{\text {rms }}$ here is the rms temperature fluctuation along a neutral surface. Mooring data cannot be used to estimate $\theta_{\text {rms }}$, because temperature variance is dominated by small vertical and horizontal scales, as we have shown in section 3 . The vertical spacing of the current meters is clearly not sufficient. Alternatively, we use CTD data. For each neutral density layer the large-

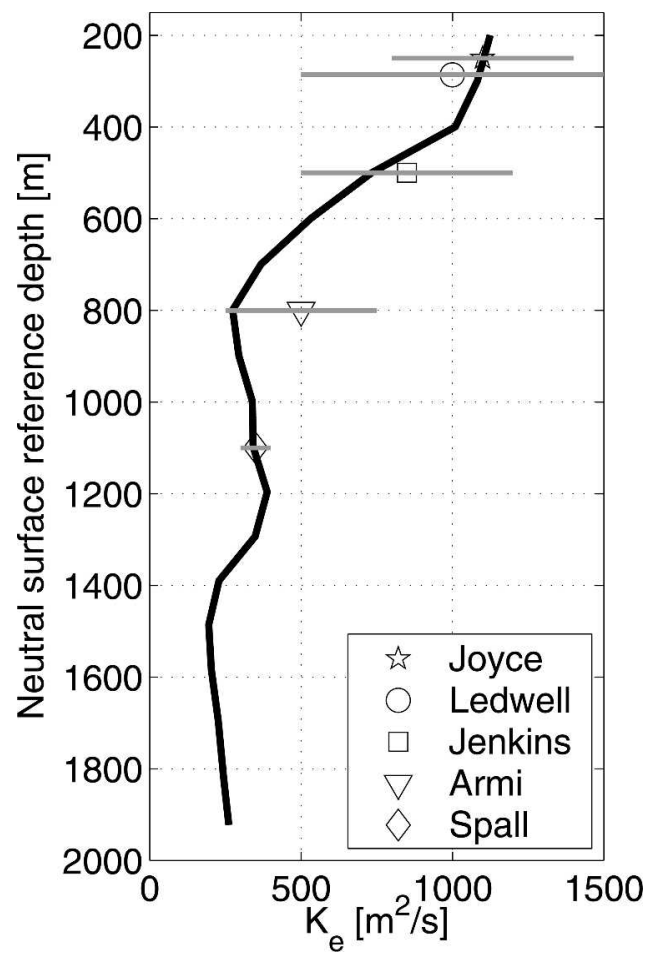

FIG. 6. Estimates of neutral diffusivities, due to mesoscale stirring along neutral density surfaces, in the NATRE region. The estimates represented with symbols correspond to the values given in Table 1. The horizontal lines are uncertainties reported by the authors. The dark line is the neutral diffusivity as estimated applying (6.3) to the NATRE dataset. 


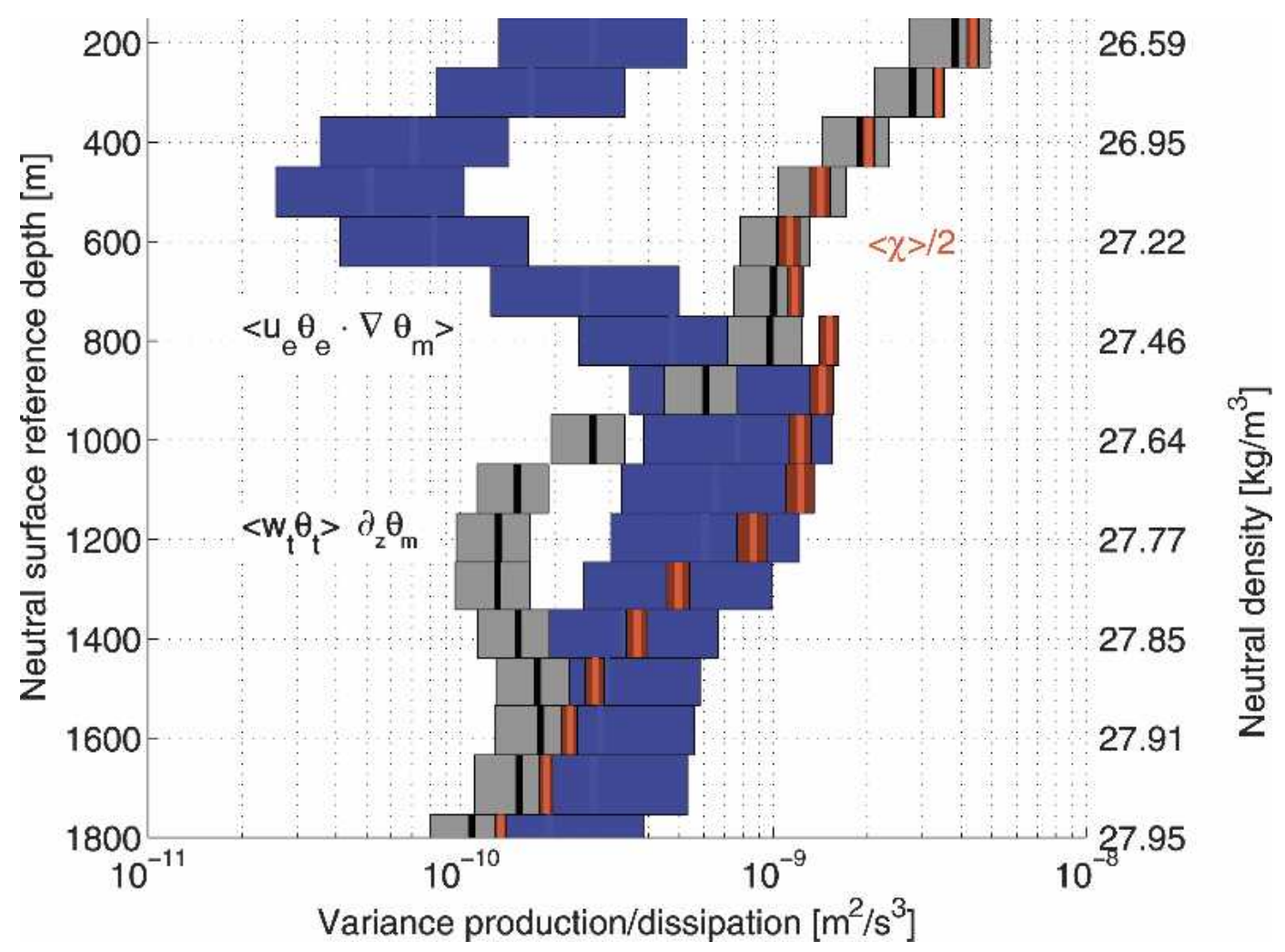

FIG. 7. Temperature variance budget analysis of the NATRE dataset. The analysis is based on the Osborn-Cox model modified to account for lateral eddy stirring. The budgets are computed for neutral layers approximately 100 $\mathrm{m}$ thick. The mean depth of each neutral surface is used as the reference vertical coordinate. Microstructure estimates of temperature variance dissipation for each layer are shown in red and the shaded boxes represent the error bars. The production of variance by turbulent motions acting on the mean diapycnal gradient is shown in black. The production of variance by eddy stirring of the mean isopycnal gradient is represented in blue. At the NACW level, temperature variance is associated with turbulence (i.e., internal wave breaking) acting on the mean diapycnal temperature gradient. At the MW level (900-1400 m), eddy stirring dominates the production of temperature gradient variance. For reference, $\mathrm{SF}_{6}$ was released by Ledwell in the density class corresponding to $300 \mathrm{~m}$.

scale temperature gradient $\left|\nabla_{n} \theta_{m}\right|$ is estimated by fitting a plane to the 100 stations in the $400 \mathrm{~km} \times 400 \mathrm{~km}$ large-scale survey grid and $\theta_{\text {rms }}$ is computed as the standard deviation of the departures from the plane fit. In the upper $800 \mathrm{~m} L_{e} \approx 200-300 \mathrm{~km}$. Below $800 \mathrm{~m} L_{e} \approx$ $100 \mathrm{~km}$. The mixing length is smaller than the survey grid, and we have some confidence that we can separate the large-scale neutral gradient from the mesoscale eddy fluctuations.

Last, we need to choose an appropriate mixing efficiency $c_{e}$. Stone (1972) used a linear analysis of baroclinic instability to suggest a value of 0.9 . Numerical simulations of a fully developed mesoscale field give values in the range of 0.01-0.4 (Visbeck et al. 1997; Haine and Marshall 1998; Karsten et al. 2002). Wunsch (1999) used a quasi-global compilation of current meters and temperature records to directly estimate temperature and velocity correlations. He found that $c_{e}$ had a global average value of 0.16 . In the following analysis we use Wunsch's value of 0.16 because it is the only data-based estimate. Resulting estimates of $K_{e}$ from (6.3) are in good agreement with previous work (Fig. 6).

In Fig. 7, we show the variance production by mesoscale eddies based on the estimates of $K_{e}$ and $\left|\nabla_{n} \theta_{m}\right|^{2}$. Errors on the large-scale gradient are estimated as $95 \%$ confidence intervals in the least squares fit used to derive $\boldsymbol{\nabla}_{n} \theta_{m}$. Uncertainties on $K_{e}$ are more difficult to estimate, because of the many heuristic arguments used in the computation. Somewhat arbitrarily we assume that the uncertainty can be as large as a factor of 2; that is, the error is $\left(K_{e} / 2,2 K_{e}\right)$, which is slightly larger than the difference between our and previous estimates at any level.

\section{b. Variance production by microscale turbulence}

In high-Reynolds-number microscale turbulence, production by shear instability mixes all tracers at the 


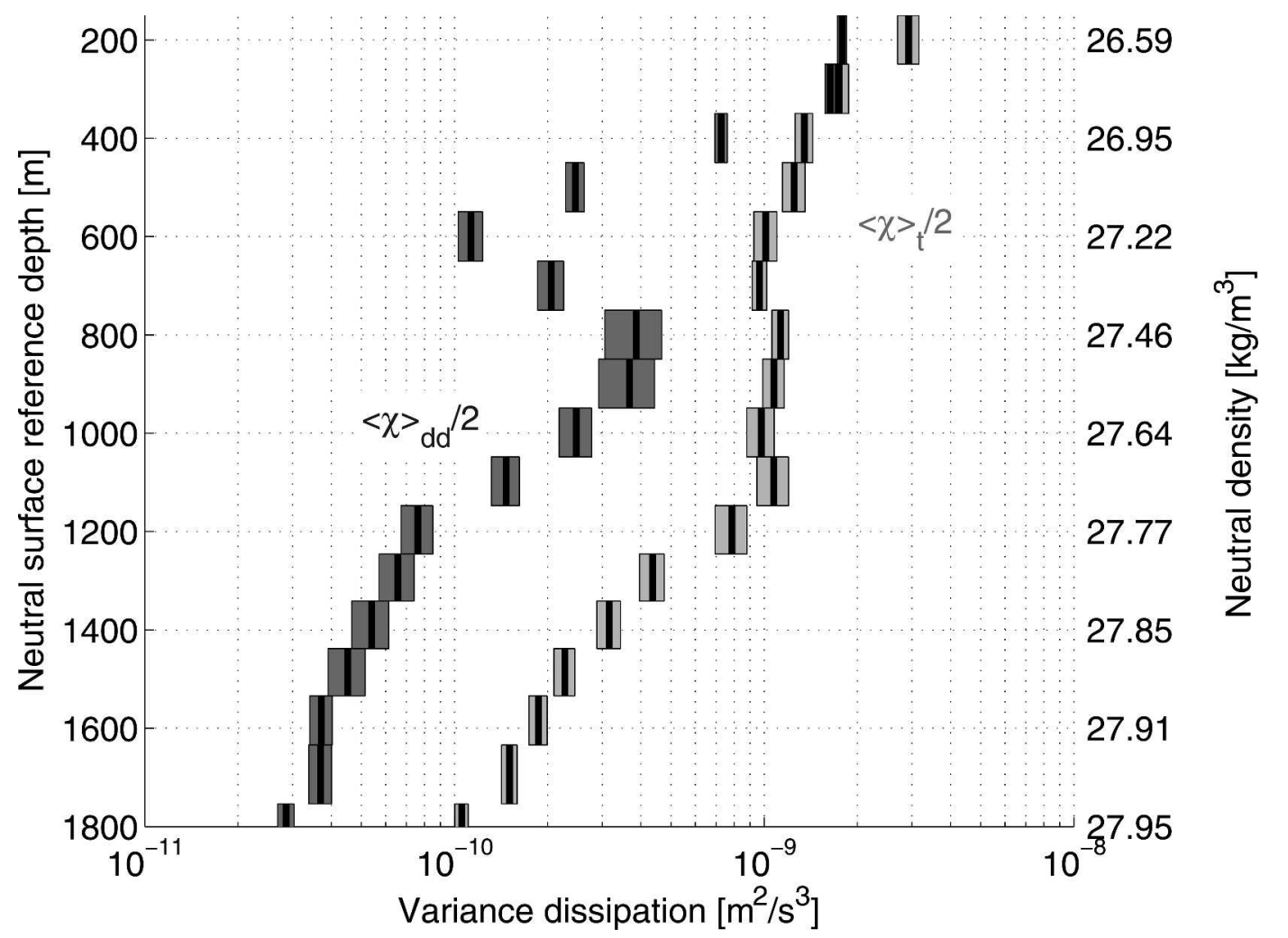

FIG. 8. Molecular dissipation of thermal variance by shear-driven turbulence (pale gray boxes) and by double diffusive convection (dark gray boxes). For each neutral density layer, mean values are shown as a vertical line, and the uncertainty as a gray box. The vertical axis is restricted to neutral density layers that did not outcrop within the survey region. The near-surface layers have much larger dissipation rates because of the enhanced turbulence generated by air-sea interactions and are not the focus of this paper.

same rate. Thus, we expect that the flux-gradient relationship for turbulent fluxes of temperature is down the mean gradient with a diapycnal diffusivity $K_{\rho}$ equal to that for buoyancy,

$$
\left\langle\mathbf{u}_{t} \theta_{t}\right\rangle \cdot \nabla \theta_{m} \approx-K_{\rho}\left(\partial_{z} \theta_{m}\right)^{2} .
$$

The turbulent generation of microscale variance is computed for each neutral surface with the same data and binning used in the analysis of the turbulent kinetic energy budget (Fig. 7). The error analysis is given in the appendix.

Salt fingers can also contribute to the generation of microscale variance. Following St. Laurent and Schmitt (1999), within each neutral density layer, the dissipation data are partitioned into two groups. All $\chi$ estimates associated with $1<R_{\rho}<2$ and $\mathrm{Ri}>1$ are presumed to be generated by salt fingers. We assume all other dissipation measurements are associated with shear-driven turbulence. In this manner we attribute some percentage of the observations to fingers $\left(P_{f}\right)$ and some to shear turbulence $\left(P_{t}=1-P_{f}\right)$. To estimate the vertical gradients of buoyancy and velocity that appear in $R_{\rho}$ and $\mathrm{Ri}$, we use the slopes of linear fits over 10-m segments, centered at the 0.5 -m intervals where $\chi$ was measured. In Fig. 8 we show the fraction of $\chi$ associated with fingers (dark gray boxes) and that associated with shear instabilities (light gray boxes). Below 400-m thermal dissipation is nearly exclusively associated with shear turbulence and fingering convection can be neglected. In the upper $400 \mathrm{~m}$, fingers account for up to half of the variance dissipation. St. Laurent and Schmitt (1999) find that fingers increase turbulent production of variance by up to $40 \%$ at these levels. Increasing our estimate of turbulent production by $40 \%$ in the upper $400 \mathrm{~m}$ would brings the turbulent production estimates (black line) to line up with the variance dissipation (red line) in Fig. 7. But this correction is minor and does not change the leading balances in the variance budgets.

The small contribution of salt fingers at the MW level might surprise some readers. MW are populated by submesoscale coherent vortices (meddies), which show up as salinity values in excess of 36.0 and are often associated with enhanced double diffusive turbulence (Ruddick and Hebert 1988). In the NATRE region 
there is no evidence of meddies and therefore the NATRE dataset might not be representative of regions closer to the Mediterranean Outflow, where meddies are frequently encountered.

\section{c. The total variance budget}

Estimates of the three terms appearing in the temperature variance budget (6.1) give quantitative support (Fig. 7) to the qualitative arguments on the role of eddies and turbulence in the NATRE region. At the NACW level, the upper $800 \mathrm{~m}$, temperature variance is produced by turbulence acting on the mean gradient and dissipated at the microscale, as assumed by the traditional Osborn-Cox model. This corresponds to the lower path in the diagram (6.6). At the MW level, there is an exchange of balance. Stirring by mesoscale eddies dominates the variance production and the leading order path of temperature variability goes through the upper branch in the diagram (6.6). The Osborn-Cox model is not appropriate at this level. Mesoscale eddies run the show. We speculate that this balance between eddy production and dissipation is typical of many regions characterized by water mass contrasts at large scales:

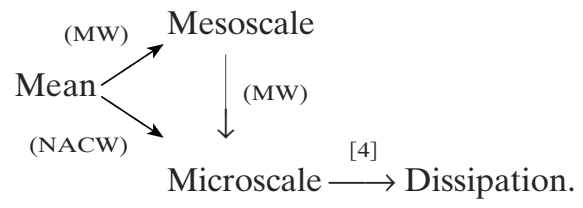

We showed that the temperature variance budget in (4.8) can be written in terms of effective diffusivities:

$$
\underbrace{K_{\rho}\left(\partial_{z} \theta_{m}\right)^{2}}_{[3]}+\underbrace{K_{e}\left|\nabla_{n} \theta_{m}\right|^{2}}_{[1]}=\underbrace{\frac{1}{2}\langle\chi\rangle}_{[4]} .
$$

The relative importance of turbulent and eddy production of temperature, that is, terms [1] and [3] in (6.7), can be quantified in terms of the ratio of isopycnal and diapycnal temperature gradients squared (Davis 1994):

$$
r=\frac{\left|\nabla_{n} \theta_{m}\right|^{2}}{\left(\partial_{z} \theta_{m}\right)^{2}}
$$

Variance production at the microscale is dominated by the mesoscale eddy stirring when $r \gg K_{\rho} / K_{e}$ and by turbulent fluxes when $r \ll K_{\rho} / K_{e}$. In Fig. 9 we show that in the upper $800 \mathrm{~m} r \ll K_{\rho} / K_{e}$ and variance is generated by the turbulent flux. At the MW level $r \gg K_{\rho} / K_{e}$ and the mesoscale flux dominates the variance production. This calculation shows that the results from the vari-

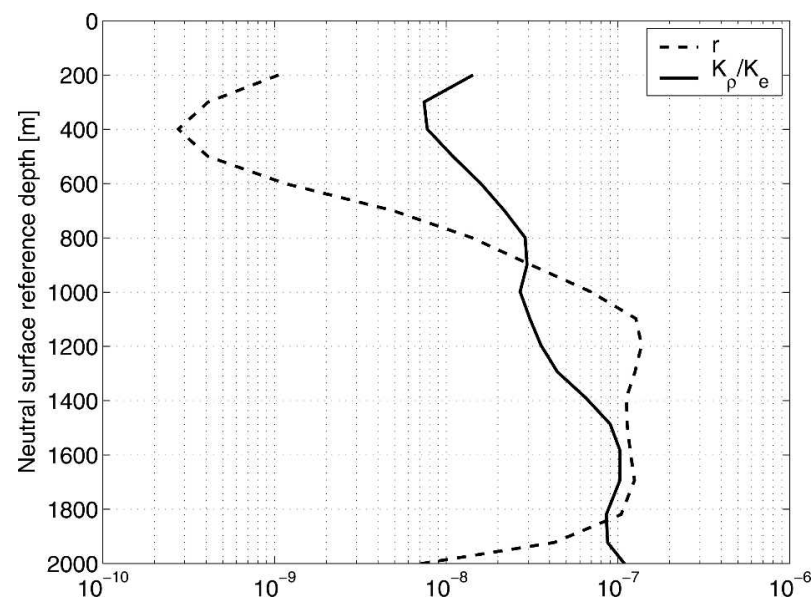

FIG. 9. Comparison of the slope squared of mean temperature surfaces $r$ (dashed line) and the ratio of diapycnal, $K_{\rho}$, and neutral, $K_{e}$, diffusivities (solid line). When the slope squared is larger than the ratio of the diffusivities, temperature variance production by mesoscale eddies dominates over turbulent production.

ance analysis are quite robust: $K_{e}$ would have to be reduced by an order of magnitude if mesoscale eddy stirring were to become subdominant in the variance budget.

We have shown that the production of variance by mesoscale and turbulent fluctuations can account for the observed temperature dissipation at all depths. This suggests that the terms neglected to derive the variance budget in (6.1) - that is, divergent and transient terms and double diffusive effects-can be at most of the same order of the production terms but not orders of magnitude larger. On the other hand, neglecting the mesoscale production term, as it is often done in the literature, can lead to an order of magnitude mismatch between estimates of variance production and dissipation.

\section{Conclusions}

In this paper we used a combination of microstructure profiles and mooring records to identify the processes that participate in the creation and destruction of thermohaline gradients in the open ocean. We analyzed data collected during the North Atlantic Tracer Release Experiment, but the results are quite general and should apply to other regions as well.

The $T-S$ relationship in the ocean interior, away from heat and haline sources, is maintained by advection, stirring, and mixing. The later two processes act very differently on $T-S$ distributions. First, stirring is dominated by mesoscale eddies with scales between $O(10-100) \mathrm{km}$, while turbulent mixing is characterized 
by motions with scales of $O\left(10^{-2}-10\right) \mathrm{m}$. Second, turbulence drives fluxes both along and across density surfaces, while mesoscale eddy motions are directed along neutral density surfaces. Third, stirring tends to increase $T-S$ variability, while turbulence mixes different water types along a straight line in $T-S$ space, the mixing line. In regions where temperature variance production is dominated by turbulence, one expects to find smooth $T-S$ profiles with wiggles only at small scales. In regions where temperature variance production is dominated by eddy stirring, one expects $T-S$ profiles to exhibit structure at the finescale.

This paradigm was used to analyze microstructure measurements from NATRE. In the thermocline (upper $800 \mathrm{~m}$ ), characterized by North Atlantic Central Waters, we found that turbulent mixing dominates and the $T-S$ relationship is very tight. In contrast, at the Mediterranean Water level (900-1400 m), the $T-S$ relationship exhibits a large degree of variability along isopycnals. This variability takes the form of sharp compensated $T-S$ gradients, that is, isopycnal $T-S$ gradients, with little signature in density. Compensated variability is generated by mesoscale stirring acting on climatological water mass contrasts along density surfaces: water mass contrasts are large at the MW level, while they are absent at the NACW level.

This interpretation of eddy stirring producing finestructure along isopycnals is confirmed quantitatively by the temperature variance budget analysis of the NATRE dataset. The analysis is based on the OsbornCox model modified to account for lateral eddy stirring, as shown graphically in the diagram (6.6). At the NACW level, temperature variance dissipation is associated with turbulence (i.e., internal wave breaking and double diffusion) acting on the mean temperature gradient. At the MW level, eddy stirring of the mean isopycnal temperature gradient is strong enough to account for all the observed thermal dissipation. Furthermore this stirring creates a local maximum in the $\langle\chi\rangle /\langle\varepsilon\rangle$ ratio. Large values of this ratio are often invoked as a signature of double diffusive intrusions (Oakey 1988). We suggest that eddy stirring can also increase this ratio.

A question arises as to what mixing processes arrest the formation of this variability in the form of compensated $T-S$ gradients. The traditional explanation is that there is some small-scale three-dimensional instability, perhaps involving vortical modes (Polzin and Ferrari 2004) and shear dispersion (Young et al. 1982; Haynes and Anglade 1997), which halts the formation of gradients before the molecular scale. This possibility can be described as passive in the sense that the small-scale mixing mechanism is independent of the $T-S$ variabil- ity, and just removes the variance produced by the stirring along density surfaces. Alternatively Garrett (1982) suggested that as the front develops, it becomes unstable to double diffusive thermohaline intrusions which limit the further narrowing of the front. This might be termed an active process in that it produces extra diapycnal mixing, that would not occur in the absence of lateral stirring. Notice that, in this scenario, eddy stirring controls the rate of double-diffusive turbulence, and hence of diapycnal mixing.

Our analysis suggests that the arrest of the $T-S$ filamentation by eddy stirring is passive at the MW level: the variance production by salt fingers is weak at those levels. Notice however that our horizontal resolution is very coarse in comparison with the scales of the $T-S$ filaments (probably between 1 and $10 \mathrm{~km}$ ). Thus it is possible that we are underestimating the high-wavenumber variance in $T-S$ gradients. We speculate that a campaign aimed at estimating $T-S$ filament widths would provide the necessary information to determine what process arrests the formation of compensated $T-S$ gradients. The idea is that the filament width $W$ would be given by a balance of mesoscale strain $\Gamma$ and turbulent mixing, i.e., $L \approx K_{t} / \Gamma$, where $K_{t}$ is the effective diffusivity of the turbulent process (see Haynes and Anglade 1997 for an expression that takes into account vertical shear as well). Expressions and dependence of the effective diffusivities on $T-S$ gradients are different for mixing due to internal wave breaking (see, e.g., Polzin 1996) and for double diffusive convection (see, e.g., Schmitt 1981; Kelley 1990). Thus the observed filament widths could be used to infer the effective diffusivity, and hence to determine the dominant turbulent mixing process.

A final remark concerns the direction of the heat, salt, and buoyancy variance cascades. The emergence of coherent structures in geophysical turbulence is typically associated with a transfer of variance from small to large scales: mesoscale fluctuations drive mean flows and ocean rings, and double diffusive instabilities produce staircases at the mesoscale (Schmitt 1994). These processes imply that the direction of the arrows in the diagram in (6.6) can be reversed. The absence of double-diffusive staircases in the NATRE dataset suggests that the upscale transfer of variance is inefficient in this region of the ocean. But in regions like the western subtropical Atlantic the presence of jets, ocean rings, and staircases might change the picture substantially. It would thus be very interesting to apply the triple decomposition presented in this paper to different regions with a more vigorous eddy field and stronger salt fingering convection. 
Acknowledgments. Ferrari and Polzin gratefully acknowledge the support of the Office of Naval Research under Award N00014-03-1-0354.

\section{APPENDIX}

\section{Estimates of Mean and Uncertainties in the Variance Budgets of Buoyancy and Temperature}

Estimating mean and variance of dissipation data is the subject of a vast literature. The apparent tendency for the statistical distributions of $\varepsilon$ (and $\chi$ ) to be lognormal has produced arguments in favor of maximum likelihood estimates (Ruddick et al. 1997). However, departures from lognormality have also been documented, and Davis (1996) concludes that arithmetic mean estimation is the most robust form of analysis. Here we adopted the arithmetic estimation for the mean. The variance of $\varepsilon$ (and $\chi$ ) are calculated using a bootstrap method (Efron 1982). The number of degrees of freedom in the bootstrap estimate is determined from the number of independent vertical segments in the ensemble. The number of independent vertical segments in the $0.5-\mathrm{m} \varepsilon$ (and $\chi$ ) data was estimated using a vertical-lag correlation analysis (St.Laurent and Schmitt 1999). The profiles are characterized by correlation scales of $5 \mathrm{~m}$ in the thermocline $(<800 \mathrm{~m})$ and 10 $\mathrm{m}$ at greater depths. A single degree of freedom is represented by the grouping of $0.5-\mathrm{m}$ data within one correlation scale in a single profile. For the ensemble of data in each neutral density class, the number of such groupings gives the total degrees of freedom.

For the uncertainty of the turbulent dissipation ratio, we have used the value estimated by St. Laurent and Schmitt (1999), $\Delta \Gamma_{t}=0.04$. In the case of $\partial_{z} b_{m}$ the uncertainty is due to variability in the mean vertical coordinate $z_{m}$ with a standard deviation of 5-10 m. These fluctuations are likely due to large-amplitude internal tides and should be included in the neutral density height averaged over the eddy scale $z_{e}$. However, the aliasing due to the 18 days over which the survey was completed does not allow a clear separation between eddy motions and temporal drifts of the mean. Conservatively we associate the full $5-10 \mathrm{~m}$ as an estimate of the uncertainty in $z_{m}$.

The standard errors of the turbulent buoyancy flux and the diapycnal diffusivity can now be calculated as follows:

$$
\begin{aligned}
\Delta\left\langle w_{t} b_{t}\right\rangle & =\left\langle w_{t} b_{t}\right\rangle\left[\left(\frac{\Delta \Gamma_{t}}{\Gamma_{t}}\right)^{2}+\left(\frac{\Delta \epsilon}{\epsilon}\right)^{2}\right] \text { and } \\
\Delta K_{\rho} & =K_{\rho}\left[\left(\frac{\Delta \Gamma_{t}}{\Gamma_{t}}\right)^{2}+\left(\frac{\Delta \epsilon}{\epsilon}\right)^{2}+\left(\frac{\Delta \partial_{z} b_{m}}{\partial_{z} b_{m}}\right)^{2}\right],
\end{aligned}
$$

where the $\Delta$ terms denote the uncertainties associated with the various fields.

The uncertainty on the estimates of turbulent temperature fluxes is given by

$$
\Delta\left(\left\langle w_{t} \theta_{t}\right\rangle \partial_{z} \theta_{m}\right)=K_{\rho}\left(\partial_{z} \theta_{m}\right)^{2}\left[\left(\frac{\Delta K_{\rho}}{K_{\rho}}\right)^{2}+2\left(\frac{\Delta h_{m}}{h_{m}}\right)^{2}\right],
$$

where $h_{m}$ is the mean thickness of a neutral density layer. We assume that uncertainties in the mean vertical gradients of temperature are associated with fluctuations in neutral density layer thickness, but not with temperature fluctuations within the layer. The alongsurface fluctuations are associated with mesoscale motions and represent a true signal included in the mesoscale variance budget.

\section{REFERENCES}

Armi, L., and H. Stommel, 1983: Four views of a portion of the North Atlantic subtropical gyre. J. Phys. Oceanogr., 13, 828857.

Batchelor, G. K., 1959: Small-scale variation of convected quantities like temperature in a turbulence fluid. Part 1. J. Fluid Mech., 5, 113-133.

Curry, R. G., 1996: Hydrobase-A database of hydrographic stations and tools for climatological analysis. Woods Hole Oceanographic Institution Tech. Rep. WHOI-96-01, 44 pp.

Davis, R. E., 1994: Diapyncal mixing in the ocean: The OsbornCox model. J. Phys. Oceanogr., 24, 2560-2576.

—, 1996: Sampling turbulent dissipation. J. Phys. Oceanogr., 26, 341-358.

Eckart, C., 1948: An analysis of stirring and mixing processes in incompressible fluids. J. Mar. Res., 7, 265-275.

Efron, B., 1982: The Jackknife, the Bootstrap and Other Resampling Plans. Society for Industrial and Applied Mathematics, $92 \mathrm{pp}$.

Garrett, C., 1982: On the parameterizations of diapyncal fluxes due to double diffusive intursions. J. Phys. Oceanogr., 12, 952-959.

—, 2001: Stirring and mixing: What are the rate controlling processes? Proc. 'Aha Huliko'a Winter Workshop, P. Müller and D. Henderson, Eds., University of Hawaii at Manoa, 1-8.

Gent, P. R., and J. C. McWilliams, 1990: Isopycnal mixing in ocean circulation models. J. Phys. Oceanogr., 20, 150-155.

Green, J. A., 1970: Transfer properties of the large-scale eddies and the general circulation of the atmosphere. Quart. J. Roy. Meteor. Soc., 96, 157-185.

Gregg, M. C., 1989: Scaling turbulent dissipation in the thermocline. J. Geophys. Res., 94, 9686-9698.

Haine, T. W. N., and J. Marshall, 1998: Gravitational, symmetric, and baroclinic instability of the ocean mixed layer. J. Phys. Oceanogr., 28, 634-658.

Hayes, S. P., H. B. Milburn, and E. F. Ford, 1984: TOPS: A freefall velocity and CTD profiler. J. Atmos. Oceanic Technol., 1, 220-236.

Haynes, P. H., and J. Anglade, 1997: The vertical-scale cascade of 
atmospheric tracers due to large-scale differential advection. J. Atmos. Sci., 54, 1121-1136.

Iselin, C. O. D., 1936: A study of the circulation of the western North Atlantic. Pap. Phys. Oceanogr. Meteor., 4, 101 pp.

_ 1939: The influence of vertical and lateral turbulence on the characteristics of waters at mid-depth. Trans. Amer. Geophys. Union, 20, 414-417.

Jackett, D. R., and T. J. McDougall, 1997: A neutral density variable for the world's oceans. J. Phys. Oceanogr., 27, 237-263.

Jenkins, W. J., 1998: Studying subtropical thermocline ventilation and circulation using tritium and He-3.J. Geophys. Res., 103, 15 817-15 831.

Joyce, T. M., 1977: A note on the lateral mixing of water masses. J. Phys. Oceanogr., 7, 626-629.

— , J. R. Luyten, A. Kubryakov, F. B. Bahr, and J. S. Pallant, 1998: Meso- to large-scale structure of subducting water in the subtropical gyre of the eastern North Atlantic Ocean. $J$. Phys. Oceanogr., 28, 40-61.

Karsten, R. H., H. Jones, and J. Marshall, 2002: The role of eddy transfer in setting the stratification and transport of a circumpolar current. J. Phys. Oceanogr., 32, 39-54.

Kelley, D., 1990: Fluxes through diffusive staircases: A new formulation. J. Geophys. Res., 95, 3365-3371.

Klein, P., A. Treguier, and B. L. Hua, 1997: Three-dimensional stirring of thermohaline fronts. J. Mar. Res., 55, 1069-1101.

Larichev, V., and I. Held, 1995: Eddy amplitudes and fluxes in a homogeneous model of fully developed baroclinic instability. J. Phys. Oceanogr., 25, 2285-2297.

Ledwell, J. R., A. J. Watson, and C. S. Law, 1993: Evidence for slow mixing across the pycnocline from an open-ocean tracerrelease experiment. Nature, 364, 701-703.

,-- , and -1998 : Mixing of a tracer in the pycnocline. $J$. Geophys. Res., 103, 21 499-21 529.

Linden, P. F., 1979: Mixing in stratified fluids. Geophys. Astrophys. Fluid Dyn., 13, 3-23.

MacVean, M. K., and J. D. Woods, 1980: Redistribution of scalars during upper ocean frontogenesis: A numerical model. Quart. J. Roy. Meteor. Soc., 106, 293-311.

Mauritzen, C., Y. Morel, and J. Paillet, 2001: On the influence of Mediterranean Water on the Central Waters of the North Atlantic Ocean. Deep-Sea Res., 48A, 347-381.

McCartney, M. S., 1977: Subantarctic Mode Water. A Voyage of Discovery: George Deacon 70th Anniversary Volume, M. V. Angel, Ed., Deep-Sea Res. Suppl., Pergamon Press, 103-119.

_ 1982: The subtropical recirculation of Mode Waters. J. Mar. Res., 40, 427-464.

Montgomery, R. B. 1938: Circulation in upper layers of southern North Atlantic deduced with use of isentropic analysis. Pap. Phys. Oceanogr. Meteor., 6, 3-55.

Moum, J. N., 1996: Efficiency of mixing in the main thermocline. J. Geophys. Res., 101, 12 057-12 069.

Nash, J. D., and J. N. Moum, 2002: Microstructure estimates of turbulent salinity flux and the dissipation spectrum of salinity. J. Phys. Oceanogr., 32, 2312-2333.

Oakey, N. S., 1988. Estimates of mixing inferred from temperature and velocity microstructure. Small-Scale Turbulence and Mixing in the Ocean, J. Nihoul and B. Jamart, Eds., Vol. 46, Elsevier Oceanography Series, Elsevier, 239-248.

Osborn, T. R., 1980: Estimates of the local rate of vertical diffusion from dissipation measurements. J. Phys. Oceanogr., 10, 83-89.
— and C. S. Cox, 1972: Oceanic fine structure. Geophys. Fluid Dyn., 3, 321-345.

Pavan, V., and I. Held, 1996: The diffusive approximation for eddy fluxes in baroclinically unstable jets. J. Atmos. Sci., 53, 1262-1272.

Polzin, K. L., 1996: Statistics of the Richardson number: Mixing models and finestructure. J. Phys. Oceanogr., 26, 1409-1425. , and E. T. Montgomery, 1996: Microstructure profiling with the High Resolution Profiler. Proc. Microstructure Sensor Workshop, Mt. Hood, OR, Office of Naval Research, 109115 .

- , and R. Ferrari, 2004: Lateral dispersion in NATRE. J. Phys. Oceanogr., 34, 247-257.

_ J. M. Toole, and R. W. Schmitt, 1995: Finescale parameterizations of turbulent dissipation. J. Phys. Oceanogr., 25, 306328.

- E. Kunze, J. M. Toole, and R. W. Schmitt, 2003: The partition of finescale energy into internal waves and subinertial motions. J. Phys. Oceanogr., 33, 234-248.

Robbins, P. E., J. F. Price, W. B. Owens, and W. J. Jenkins, 2000: The importance of lateral diffusion for the ventilation of the lower thermocline in the subtropical North Atlantic. J. Phys. Oceanogr., 30, 67-89.

Rohr, J. J., E. C. Itsewire, and C. W. VanAtta, 1984: Mixing efficiency in stably-stratified decaying turbulence. Geophys. Astrophys. Fluid Dyn., 29, 221-236.

Ruddick, B., and D. Hebert, 1988. The mixing of Meddy "Sharon." Small-scale Mixing in the Ocean, J. C. J. Nihoul and B. M. Jamart, Eds., Vol. 46, Elsevier Oceanography Series, Elsevier, 249-262.

— D. Walsh, and N. Oakey, 1997: Variations in apparent mixing efficiency in the North Atlantic Central Waters. J. Phys. Oceanogr., 27, 2589-2605.

Schmitt, R. W., 1981: Form of the temperature-salinity relationship in the central water: Evidence for double-diffusive mixing. J. Phys. Oceanogr., 11, 1015-1026.

_ 1994: Double diffusion in oceanography. Annu. Rev. Fluid Mech., 26, 255-285.

—, J. M. Toole, R. L. Koehler, E. C. Mellinger, and K. W. Doherty, 1988: The development of a fine- and microstructure profiler. J. Atmos. Oceanic Technol., 5, 484-500.

Schott, F., and H. Stommel, 1978: Beta spirals and absolute velocities in different oceans. Deep-Sea Res., 25A, 961-1010.

Siedler, G., A. Kuhl, and W. Zenk, 1987: The Madeira Mode Water. J. Phys. Oceanogr., 17, 1561-1570.

Spall, M. A., P. L. Richardson, and J. Price, 1993: Advection and eddy-mixing in the Mediterranean Salt Tongue. J. Mar. Res., 51, 797-818.

St. Laurent, L., and R. W. Schmitt, 1999: The contribution of salt fingers to vertical mixing in the North Atlantic Tracer Release Experiment. J. Phys. Oceanogr., 29, 1404-1424.

Stommel, H., 1979: Determination of water mass properties pumped down from the Ekman layer to the geostrophic flow below. Proc. Natl. Acad. Sci. U.S.A., 76, 3051-3055.

Stone, P. H., 1972: A simplified radiative-dynamical model for the static stability of rotating atmospheres. J. Atmos. Sci., 29, 405-418.

Sverdrup, H. U., M. W. Johnson, and R. H. Fleming, 1942: The Oceans: Their Physics, Chemistry, and General Biology. Prentice-Hall, 1087 pp.

Toole, J. M., K. L. Polzin, and R. W. Schmitt, 1994: New estimates 
of diapyncal mixing in the abyssal ocean. Science, 264, 11201123.

Visbeck, M., J. Marshall, T. Haines, and M. Spall, 1997: On the specification of eddy transfer coefficients in coarse-resolution ocean circulation models. J. Phys. Oceanogr., 27, 381-402.

Wunsch, C., 1997: The vertical partition of oceanic horizontal kinetic energy. J. Phys. Oceanogr., 27, 1770-1794.

1999: Where do ocean heat fluxes matter? J. Geophys. Res., 104, 13 235-13 249.
Wüst, G., 1935: Schichtung und Zirkulation des Atlantischen Ozeans-II Lieferung. DieStratosphare. Wiss. Ergebn. dt. atlant. Exped. Meteor., 6, 109-288.

Young, W. R., P. B. Rhines, and C. J. R. Garrett, 1982: Shear-flow dispersion, internal waves and horizontal mixing in the ocean. J. Phys. Oceanogr., 12, 515-527.

Zang, X., and C. Wunsch, 2001: Spectral description of lowfrequency oceanic variability. J. Phys. Oceanogr., 31, 30733095 . 